\title{
Juventud, ciudadanía y responsabilidad social ${ }^{1}$
}

\author{
Guillermo Williamson Castro \\ Tamara Torres Huechucura ${ }^{3}$ \\ Jorge Villenas Molina ${ }^{4}$
}

La juventud vive siempre en trance de heroísmo. Es desinteresada, es pura. No ha tenido tiempo aún de contaminarse. No se equivoca nunca en la elección de sus propios maestros. Ante los jóvenes no se hace mérito adulando o comprando. Hay que dejar que ellos mismos elijan sus maestros y directores, seguros de que el acierto ha de coronar sus determinaciones. En adelante solo podrán ser maestros en la futura república universitaria los verdaderos constructores de alma, los creadores de verdad, de belleza y de bien.

La juventud argentina de Córdoba a los hombres libres de Sud América Manifiesto de la Federación Universitaria de Córdoba - 1918

\section{RESUMEN}

En este trabajo empleamos el enfoque crítico para discutir el papel de los jóvenes como actores sociales responsables del desarrollo de sus territorios y de la transformación de la sociedad, es decir, desde la sociedad local y nacional en que viven, crecen y se desarrollan. Este enfoque lo articulamos con tres marcos de definiciones principales: la educación y pedagogía crítica, la formación ciudadana según la Ley 20.911 y el desarrollo humano según el Programa de Naciones Unidas para el Desarrollo (PNUD). Muchas de las grandes transformaciones culturales de los siglos XX y XXI han sido impulsadas por movilizaciones juveniles cuya condición de colectivo generacional abusado, disconforme, vulnerado, creativo, apasionado, les

1 Artículo recibido el 09/09/2018. Artículo aprobado el 03/12/2018.

2 Chileno, Licenciado en Educación, Académico e investigador Universidad de La Frontera (UFRO) Temuco, Chile. E-mail: guillermo.williamson@ufrontera.cl

3 Chilena, Socióloga, Académico e investigador Universidad de La Frontera (UFRO) Temuco, Chile.E-mail: t.torres@ufromail.cl

4 Chileno, Profesor de estado en historia, geografía y educación cívica. Académico e investigador Universidad de La Frontera (UFRO) Temuco, Chile. E-mail: Jorge. villenas@ufrontera.cl 
imprime una energía transformadora de la sociedad o, al menos, de la educación en Chile. Reconociendo la gran heterogeneidad existente al interior de este segmento social, que nos obliga a hablar de "juventudes", hay un sector que se asume como actor social y/o político, individual y/o colectivo, como ciudadano responsable a través de alguna expresión específica de la construcción y transformación de la sociedad. Este es el grupo del que trata este texto, como realidad y/o como deseo.

Palabras clave: Jóvenes, cambio social, educación, pedagogía crítica.

\section{Youth, citizenship and social responsibility}

\section{ABSTRACT}

In this paper we use a critical approach in order to discuss the role of young people as social actors responsible for the development of their territories and the transformation of society, that is, from the local and national society in which they live, grow and develop. This approach is articulated with three main definition frameworks: education and critical pedagogy, citizen education according to Law 20,911 and human development according to the United Nations Development Program (UNDP.) Many of the great cultural transformations of the 20th and 21st Centuries have been driven by youth mobilizations with a condition of generational collective abused, dissatisfied, violated, creative, passionate, imprinting a transforming energy for society or, at least, for Chilean education. Recognizing the great heterogeneity existing within this social segment forcing to speak of "youth," there is a sector that assumes itself as an individual and/or collective social and/or political actor, as a responsible citizen through some specific expression of the construction and transformation of society. This text is focused in that group, as a reality and/or as a desire.

Keywords: Young people, social change, education, critical pedagogy

\section{Juventude, cidadania e responsabilidade social}

\section{RESUMO}

Neste trabalho empregamos a perspectiva crítica para discutirmos o papel dos jovens como atores sociais responsáveis do desenvolvimento de seus territórios e da transformação da sociedade, quer dizer, desde a sociedade local e nacional em que vivem, crescem e se desenvolvem. Esta perspectiva a articulamos com três marcos de definições principais: a educação e pedagogia crítica, a formação cidadã segundo a Lei 20.911 e o desenvolvimento humano segundo o Programa das Nações Unidas para o Desenvolvimento (PNUD). Muitas das grandes transformações culturais do Século XX e XXI têm sido impulsadas por mobilizações juvenis cuja condição de coletivo geracional abusado, desconforme, vulnerado, criativo, apaixonado, lhes imprime uma energia transformadora da sociedade ou, ao menos, da educação no 
Chile. Reconhecendo a grande heterogeneidade existente ao interior deste segmento social que os obriga a falarem de "juventudes", há um setor que se assume como ator social e/ou político, individual e/ou coletivo, como cidadão responsável através de alguma expressão específica na construção e transformação da sociedade. Este é o grupo do que trata este texto, como realidade e/ou como desejo.

Palavras-chave: Jovens, mudança social, educação, pedagogia crítica

\section{Introducción ${ }^{5}$}

Una de las cuestiones sociales emergentes de la modernidad, desde la Revolución Industrial, pasando por los siglos XIX y XX, es el reconocimiento visible a las generaciones: los niños y los jóvenes dejan de ser invisibles, de ser considerados pequeños adultos o una población en una etapa de minoridad dependiente durante el proceso de hacerse persona: pasan a constituirse en un grupo social y trabajador que transita entre la infancia y la adultez, en construcción de su inserción social e identidad, que se relaciona de modo particular con el Estado, la escuela, la familia, el ejército, la justicia (Adorno, 2001). Este aparecimiento reciente en la historia de la humanidad de la infancia, la adolescencia, la juventud, ha ido progresivamente acompañado de dos fenómenos complementarios y contradictorios al mismo tiempo: por una parte, se constituyen en sujetos de derechos humanos, individuales y colectivos y, por otro, en sujetos consumidores objeto de procesos de producción específicos. Junto a los derechos de la infancia y la adolescencia, que los constituye en sujetos activos protegidos por la legislación nacional y universal, el mercado capitalista los convierte en un colectivo social potencialmente demandante de productos tangibles e intangibles, por lo que orienta parte de sus procesos productivos y de acumulación de capitales a este segmento poblacional. Una característica de la juventud es su vida en medio

5 Este texto es parte del Programa de Formación Ciudadana y de la investigación "Desarrollo de los Planes de Formación Ciudadana en las Comunidades Educativas de un Territorio" que lleva a cabo el Departamento de Educación de la Universidad de La Frontera (2016-2018), con el apoyo del Ministerio de Educación (Res. Ex. 2927, 07.06.2017). 
de contradicciones y ambigüedades, incertezas e inseguridades, mientras buscan diferenciarse de niños y adultos y encontrar su lugar en la sociedad y en sus círculos de referencia afectivos, sociales, culturales y generacionales.

En la sociedad actual, la juventud -que es el grupo social al que se orienta este texto- es objeto de múltiples investigaciones, artículos, publicaciones, tanto desde los estudios de mercado (por su potencial consumidor), de los conservadores y de seguridad nacional (por su potencial rebelde), del pensamiento crítico (por su potencial transformador), debido a su emergencia como colectivo social que conforma una identidad y "cultura", que irrumpe superando fronteras culturales, ideológicas, lingüísticas y estéticas, lo que se ha extendido exponencialmente con el desarrollo y ampliación de diversas plataformas de redes sociales.

En este último enfoque -crítico- nos situamos para discutir el papel de los jóvenes como actores sociales responsables del desarrollo de sus territorios y de la transformación de la sociedad, es decir, desde la sociedad local y nacional en que viven, crecen y se desarrollan. Este enfoque lo articulamos con tres marcos de definiciones principales: la educación y pedagogía crítica, la formación ciudadana según la Ley 20.911 y el desarrollo humano según el Programa de Naciones Unidas para el Desarrollo (PNUD). Partimos de una observación en las grandes transformaciones culturales de los siglos XX y XXI: muchas han sido impulsadas por grandes movilizaciones juveniles que, con la fuerza de su generosidad y la intuición-convicción de que más que una cuestión de clases es su condición de colectivo generacional abusado, disconforme, vulnerado, creativo, apasionado lo que genera la energía transformadora de la sociedad o, al menos, de la educación ${ }^{6}$.

6 En Chile puede reconocerse el papel de la juventud en la construcción temprana de partidos políticos, su importante institucionalización como sus juventudes, la reforma universitaria de 1967, el movimiento "hippie" de la década del sesenta del siglo XX, el de las juventudes católicas progresistas; al mismo tiempo, la gran participación juvenil -especialmente poblacional, social, cultural y política- en la lucha contra la dictadura cívico-militar (1973-1990), el movimiento de estudiantes 
La juventud aún no encuentra un espacio propio, único y colectivo de identidad y representación, sino que se caracteriza por la diversidad de expresiones sociales y culturales que la constituye como generación. En estricto sentido, no se podría hablar de "la juventud" sino de "juventudes", en plural, no solo por su extensión planetaria multicultural, sino por su diversidad en cada sociedad, como colectivos informales subculturales o grupos de interés, con sus propias creencias, estéticas, estilos de vida, comportamientos, que les otorgan una identidad particular en el conjunto de la cultura hegemónica (por ejemplo, las llamadas "tribus urbanas"7 o grupos que comparten intereses comunes orientados a lo público). Sin embargo, estas subculturas pueden articularse y reconocerse a nivel global a través de las redes sociales y otras plataformas de comunicación global (por ejemplo, los skaters los punk, los góticos, los hippies, los raperos, se reconocen en cualquier país).

Hay una juventud excluida e incluso anómica: en pobreza, al borde de los riesgos de la muerte, la enfermedad, el hambre, la drogadicción, el desempleo y la consecuente desesperanza en la posibilidad de una vida mejor, o descreencia en una sociedad libre que les permita desarrollar sus potencialidades; son jóvenes - muchos de los cuales viven en medio de la violencia institucional o física-, sin acceso al ejercicio de los derechos de los que son sujetos formales, sin expectativas, más allá de su condición de sujetos de consumo de drogas ilícitas o productos de los intercambios ilegales del mercado, sin oportunidades del sistema capitalista para ellos, ni posibilidades de integración social propositiva, contributiva, efectiva a una comunidad que les debiera acoger.

Hay una juventud, que no excluye a miembros de los grupos anteriores, que se asume como parte de un movimiento social transformador, con diversidad de ideas políticas y sociales, asociada a

secundarios (llamado "de los pingüinos"), el universitario y el feminista del inicio del siglo XXI, que han impactado en la legislación educacional y en el marco cultural del país.

7 Ver el sitio: https://www.lifeder.com/tribus-urbanas/ 
juventudes político-partidarias o colectivos sociopolíticos, de una u otra forma a procesos pedagógicos o educacionales de educación popular o de movimientos estudiantiles secundarios o universitarios. Un caso paradigmático en América Latina fue el movimiento de estudiantes secundarios del año 2011, que movilizó a la educación secundaria -e incluso básica- por un conjunto de demandas educacionales de larga data y la consecución de objetivos propios del ejercicio de los derechos educacionales que deberían estar asegurados para ellos (Donoso y Dragnic, 2015). Hay un segmento que se asume como actor social o político, individual o colectivo, como ciudadano, responsable a través de alguna expresión específica de la construcción y transformación de la sociedad. Este es el segmento del que trata este texto, como realidad y/o deseo.

A inicios de 2016 se promulga la Ley 20.911 de Formación Ciudadana orientada al sistema escolar y la formación inicial de profesores; el Ministerio de Educación impulsa un programa de difusión social, instalación en el sistema educativo y formación docente, referido a la formación ciudadana en acuerdo con universidades públicas. Recogeremos de ella uno de los ejes de esta publicación. En el primer acápite de este texto contextualizamos la situación de los jóvenes respecto de su opinión sobre su condición ciudadana, social y política. Un segundo eje analiza la Ley 20.911 desde la perspectiva educacional y la formación inicial docente, que fundamenta la responsabilidad social en la construcción y desarrollo de su propia vida y de una sociedad democrática y libre ${ }^{8}$. Un tercer eje trata de la participación y responsabilidad de jóvenes en relación al desarrollo humano y local de sus territorios de origen para el ejercicio de la ciudadanía ${ }^{9}$. Finalmente, a partir de estas reflexiones, proporcionamos algunas pistas que puedan contribuir a formular políticas y programas, públicos y de la sociedad

8 Para ello nos apoyaremos en información recogida del trabajo con liceos HumanistaCientíficos y Técnico-Profesionales, y en la Educación de Personas Jóvenes y AdultasEPJA, en el Programa de Formación Ciudadana MINEDUC/UFRO que se lleva a cabo en La Araucanía (2016-2018).

9 Este eje se apoya en la Tesis de Magister en Desarrollo Humano Local y Regional de Torres (2017). 
civil, adecuados a los territorios locales y regionales, que es la escala territorial que nos interesa en este trabajo.

\section{La juventud y las juventudes}

Las definiciones de "juventud" son construcciones sociohistóricas que se refieren a una etapa de la vida humana marcada por hitos y acontecimiento importantes para el desarrollo personal y social, es decir, dependen de los territorios, culturas y época de definición, se encuentran en la literatura socioeducativa o sociológica que consideran la edad como un determinante central, es decir, utilizan una cohorte de rangos en el proceso de desarrollo humano a partir de una serie de consideraciones sociales históricas que asumen dimensiones físicas, psicológicas, pero, sobre todo, vinculadas a su inserción en la sociedad, a través de la educación, el empleo, las fuerzas armadas, la responsabilidad social y los derechos específicos. Los jóvenes son, según la definición de las Naciones Unidas, las personas con edades comprendidas entre los 15 y los 24 años de edad. La UNESCO entiende que los jóvenes constituyen un grupo heterogéneo, en constante evolución y que la experiencia de "ser joven" varía mucho según las regiones del planeta e incluso dentro de un mismo país"10. Las leyes educacionales de Chile aseguran el derecho a la educación hasta el IV año de enseñanza media (aproximadamente a los 18 años) ${ }^{11}$, la obligación del Estado de asegurar oportunidades de educación hasta los 21 años $^{12} \mathrm{y}$, en el caso de las personas con necesidades educativas especiales, hasta los $26^{13}$, es decir, la educación, como compromi-

10 Ver el sitio: http://www.unesco.org/new/es/popular-topics/youth/

11 La educación básica y la educación media son obligatorias, debiendo el Estado financiar un sistema gratuito destinado a asegurar el acceso equitativo, inclusivo y sin discriminaciones arbitrarias a ellas de toda la población, así como generar las condiciones para la permanencia en el mismo de conformidad a la ley (https://www. ayudamineduc.cl/ficha/derecho-la-educacion-y-libertad-de-ensenanza-10).

12 El 22 de mayo de 2003 se dictó la Ley $\mathrm{N}^{\circ}$ 19.876, que extendió la obligatoriedad y, por ende, el financiamiento estatal gratuito a la educación media con un límite de edad de 21 años (Flores, 2014).

13 Ley $\mathrm{N}^{\circ} 20.422$ (03.12.2010, publicada el 10.02-2018, modificada el 23.05.2018 en la Ley $\mathrm{N}^{\circ}$ 21.089). Ver el sitio: https://www.leychile.cl/Navegar?idLey=20422\&r=1 
so del Estado con la formación de las generaciones más jóvenes, abarca al menos entre los 0 y 26 años.

El Diccionario de la Real Academia Española la define como: "periodo de la vida humana que precede inmediatamente a la madurez", "primeros tiempos de algo", "energía, vigor, frescura"14.

Definimos “juventud” según el criterio institucional del Instituto Nacional de la Juventud: entre 15 y 29 años (INJUV, 2018a). La 8 . Encuesta Nacional de Juventud 2015 muestra un diagnóstico completo de lo que caracteriza la juventud chilena; del conjunto de indicadores hemos seleccionado aquellos relevantes para efectos de este texto.

El 50,8\% son hombres y el 49,2\% mujeres; el 30,9\% tiene entre 15-19 años, el 34,1\% entre 20-24 años y un 35\% entre 25-29 años, es decir, un $65 \%$ tiene 24 años o menos: un alto porcentaje se encuentra en el rango de definición de UNESCO. El 86,7\% se define como urbano y un 13,3\% rural. El 9,8\% declara pertenecer a un pueblo indígena, de ese porcentaje el $80 \%$ se considera mapuche ${ }^{15}$. Un $96,1 \%$ se declara chileno, un $1 \%$ con doble nacionalidad (chilena y otra) y un $2,8 \%$ extranjero (otra nacionalidad). En la educación formal participa un $49,1 \%$, con una escolaridad promedio de 12,4 años lo que indica estudios al menos de 12 años: el derecho constitucional a la educación. Un 70,9\% vive en casa de sus padres, un $8,8 \%$ en casa de familiares y un $13,6 \%$ en casa propia o arrendada. Un $31,3 \%$ es padre o madre. Su participación laboral es de un 35,2\%, bajo el promedio de América Latina que es de un $47,6 \%$, lo que podría explicarse por la mayor permanencia en el sistema educacional y la baja tendencia a combinar estudio y trabajo de los jóvenes (INJUV, 2018b). La pobreza, entre los 18 a 29 años, es de un 11,0\% (CASEN, 2015).

14 Ver el sitio: http://dle.rae.es/?w=juventud

15 En La Araucanía el porcentaje de total de jóvenes que se declara indígena es un 31,1\% y en Arica-Parinacota un 32,3\%. 
Estos son promedios que esconden diferencias internas a las categorías pero que contribuyen a conformar una imagen global de la diversidad de la juventud actual: de género, etaria, de nacionalidad, de pertenencia a pueblos indígenas, de ejercicio de derechos educacionales, laborales, culturales, sociales. Más que juventud, hay juventudes. Esta diversidad expresa que su relación con la democracia, la ciudadanía y la construcción de su constitución como sujeto de derecho, es variada, multifactorial, con unidad como generación etaria pero diversa en sus identidades: como es la juventud.

Desde los derechos y prácticas de inclusión y participación social y política (2015): un 20,7\% se interesa en la política, aunque un $36,6 \%$ conversa de ella con otros jóvenes. Se informan principalmente por la televisión (62,7\%), aunque un $38,8 \%$ señala que las redes sociales sirven para informarse y un $21,1 \%$ para incidir en política. La satisfacción con la democracia es de un $12 \%$ y la insatisfacción de un $42 \%$; sin duda que para el desarrollo de un sistema democrático es un indicador de riesgo, sobre todo si se relaciona con que solo un $44,3 \%$ considera que la democracia es preferible a cualquier forma de gobierno, incluso un $15,5 \%$ señala que, en ciertas circunstancias, un gobierno autoritario es mejor que uno democrático y un $17,8 \%$ que le da lo mismo el régimen que sea: es decir un 33,3\% no valora la democracia en sí misma. A nivel nacional, un 55,9\% señaló que no fue a votar en la primera vuelta presidencial de 2013 (INJUV, 2018a).

Los datos parecen mostrar que la juventud actual no tendría arraigada una conciencia de ciudadanía como participación responsable, en la construcción de una sociedad democrática como la que conocen u otra, sea que se identifique como una sociedad de oportunidades (versión individual o liberal) o como una sociedad participativa de derechos (versión de fundamento social o socialista). ¿Por qué? ¿Es tan así?

La democracia representativa se encuentra en crisis general, la sociedad civil exige una democracia más participativa. Esta crisis, en 
el marco de la modernización capitalista neoliberal, adopta modalidades contradictorias en su propio seno, producto de su incertidumbre y ambivalencia estructural y sistémica, generando desafección institucional, especialmente respecto de los partidos políticos, y buscando nuevas formas de participación: sociales, de diversión, políticas, aunque no institucionales (Benítez y Echeverría, 2018). Un sondeo telefónico del INJUV, antes de las elecciones de 2017, indicaba que un $44 \%$ de los jóvenes tiene un sentimiento de desconfianza respecto de la política, a pesar de que un $71 \%$ considera que las votaciones son un factor de cambio en la sociedad (INJUV, 2017). Algo central parece ser la construcción de organizaciones en torno a intereses comunes más que al bien común colectivo, el que, bajo esta premisa, sería resultado de acciones tanto de políticas estructurales (sobre las cuales se desconfía) como de un agregado de acciones de organizaciones propias, en torno a intereses particulares con identidad y libertad de gestión.

Los medios de comunicación aparecen más importantes para la información juvenil ciudadana que las redes sociales, aunque éstas tienen un papel relevante para los jóvenes, en su formación de opinión, conciencia social y política. La ciudadanía es una tarea urgente por construir, asociada necesariamente a la valorización de la democracia como sistema político, social y cultural, que combina responsabilidad con el mundo compartido con otros, el ejercicio de derechos, en una sociedad de libertad y diversidad con igualdad.

Los datos nos llevan a poner el foco no solo en los jóvenes y los medios de comunicación, sino en la sociedad y su institucionalidad. Respecto del concepto de "ciudadanía" y su relación con la juventud, es necesario reconocer los límites de la visión clásica y las concepciones vigentes en la subjetividad juvenil como en sectores sociales que luchan por superar las visiones formales que definen lo que es y no es ser ciudadano(a):

Una primera que remite a la creencia que los niños, niñas y adolescentes NO son ciudadanos hasta que lleguen a la mayo- 
ría de edad, es decir, los 18 años en el caso de nuestro país. Esta referencia de ciudadanía dice relación directa con la condición legal de una persona; principalmente, con su nacionalidad y su derecho a elegir y ser elegido en las elecciones democráticas. Por esta razón, se argumenta que los niños no podrían ser ciudadanos antes de ejercer esas condiciones legales. La segunda, reconoce que los niños, niñas y adolescentes son ciudadanos. Entendiendo como ciudadanía el derecho a tener derechos y ejercerlos independiente de su condición social, jurídica, cultural, política de religión o etnia. Agregándose que la condición de ciudadano es dada, pero también constantemente recreada y formada. (Oyarzún, et al., 2008, p. 57)

La participación con identidad para la gestión social es la cuestión central para el ejercicio de una ciudadanía juvenil. El Consejo de Europa (2017) propone el "Compass, Manual para la Educación en los Derechos Humanos (EDH) con jóvenes", que plantea ocho niveles de participación, que van desde la manipulación a la toma de decisiones compartida ${ }^{16}$. Williamson (2002) señala que la participación se mueve en un rango entre la consulta (mínimo) y la autogestión (máximo), con diversos grados de gestión participativa entre ambos polos. La ciudadanía no es una sola expresión de conciencia y práctica social, sino que para los jóvenes, en una sociedad capitalista, adulta, centralizada, con educación sexista y clasista vertical, con una democracia en la que permanecen rasgos autoritarios, concentradores y segregadores, es un desafío de construcción política y social, pero también una responsabilidad para la educación en sus diversas expresiones y modalidades y en particular del sistema escolar. Avanzar de una participación manipulada a una de toma de decisión compartida, o de una de consulta a una de autogestión, supone formación ciudadana: ense-

16 Escalón 8: toma de decisiones compartida; Escalón 7: iniciados y dirigidos por jóvenes; Escalón 6: iniciados por adultos, comparten las decisiones con los jóvenes. Escalón 5: jóvenes consultados e informados. Escalón 4: jóvenes asignados pero informados. Escalón 3: falsa integración de los jóvenes. Escalón 2: decoración. Escalón 1: manipulación (Council of Europe, 2017, pp. 7-8). 
ñar las virtudes exigidas para vivir y construir de modo colectivo una vida buena para todos, sin dejar de asumir las contradicciones sociales y las diferencias que existen en la sociedad (Argumedo, 2011).

Una sociedad buena resulta de buenos ciudadanos y estos deben y pueden ser formados en la escuela, institución donde los profesores activos y en formación juegan o jugarán un papel central y crítico.

\section{Formación ciudadana y Ley 20.911 (2016). Algunos apuntes pensando en la juventud}

A partir de marzo de 2016 se dio inicio a la puesta en marcha de una nueva política pública para el sistema educativo chileno, referida a la creación de planes de formación ciudadana para todos los establecimientos educacionales reconocidos por el Estado, los cuales, en todos sus niveles -parvularia, básica y media-, deben elaborar un documento que exteriorice y oriente las prácticas en esta línea y que complemente el currículum escolar. Es la ley 20.911 (2016) que instala el enfoque de formación ciudadana en la educación chilena, a partir de un plan educativo que debe entregar a los estudiantes los lineamientos necesarios para su formación como personas integrales, que asuman una vida responsable para una sociedad libre, se formen en valores de libertad y justicia social y como ciudadanos, cuenten con una visión de mundo puesta en el ser humano en su conjunto, en sus relaciones sociales y territoriales, factores básicos para la convivencia democrática.

Esta prescripción responde a los nuevos escenarios políticos globales, en relación a la sentida necesidad de ampliar y diversificar los procesos formales de participación ciudadana, relacionados con las crecientes demandas de espacios de intervención y decisión social y política, que incida en los destinos de los gobiernos, aspiraciones que van más allá de la sola reivindicación de la importancia del sufragio. Un ejemplo es lo que ha pasado en los úl- 
timos años en Chile, a partir de las diferentes formas de acción de movimientos ciudadanos, que se han movilizado ante el Estado y sus autoridades políticas para exigir el mejoramiento de las condiciones mínimas de subsistencia para todos los habitantes del país, principalmente en lo referido a la seguridad social (v.gr. Movimiento "No Más AFP") o a las formas de acceso, financiamiento y de organización en la educación superior.

Estas reivindicaciones expresan una voluntad colectiva ciudadana que, de cuando en cuando, se manifiesta dando cuenta de un espíritu asociativo y solidario que hace frente a la política institucional estatal (PNUD, 2016). En ese contexto, los jóvenes requieren de un sistema educativo que no solo tenga una mirada técnicoacadémica vinculada a lo que tradicionalmente se ha entendido por educación cívica, con un enfoque puesto en las instituciones y marcos regulatorios de la sociedad, en la reproducción de conocimiento sobre el Estado, sus poderes normativos institucionales y el funcionamiento del sistema político, sino que en un enfoque de carácter integral, no solo para abarcar el contenido de lo netamente cívico, sino para que focalice la formación integral de la persona humana, en cuanto ciudadano, con valores que se reflejen en el fortalecimiento de la democracia como sistema político y, también, como forma de vida fundada en valores de responsabilidad, justicia social y solidaridad.

Debido a los desafíos impuestos por un escenario internacional que provoca cambios vertiginosos, que enfrenta nuevas reivindicaciones y retos desde los movimientos ciudadanos, es que los jóvenes requieren con urgencia ser imbuidos en principios éticos que afecten fuertemente su proceso de maduración cognitivo y social (Flanagan y Levine, 2015; Diccionario de Ciencias de la Educación, 2003). La formación ciudadana es una propuesta pedagógica que potencia el desarrollo psicológico de los estudiantes, en orden a cultivar, desde la escuela, prácticas políticas que contribuyan al desarrollo de la responsabilidad social formando ciudadanos cívicamente competentes (MINEDUC, 2013; 2016a; 2016 b), contribuyendo al fortalecimiento del proceso de madura- 
ción y del perfil psicológico de los jóvenes, repercutiendo en la valoración del sistema democrático y de su promoción y aplicación en la sociedad en su conjunto.

Por ello, una de las ideas finales de la ley en comento prescribe que, pensando en la educación ciudadana de los jóvenes en el sistema escolar, se deberá impulsar la ciudadanía también en la formación inicial docente, cuestión que representa un desafío para las instituciones de educación superior que se especializan en esta área de la educación. Ciertamente, en este escenario educativo se debe reconocer la responsabilidad social que tiene el profesorado en orientar a sus estudiantes en los principios de la ciudadanía como expresión de un sujeto social activo, autónomo y con conciencia de su responsabilidad social. Son ellos los que deben poseer las competencias necesarias para generar una relación con sus educandos, a fin de orientarlos en los principios de una ciudadanía crítica, activa y responsable con el ejercicio y las consecuencias sociales de sus derechos y deberes, la cual no solo debe concebirse en términos cognitivos, sino como una forma de vida, de manera de canalizar la orientación planteada por esta misma ley. Es prepararlos para llevar una vida responsable que sirva de fundamento para la vida democrática del país, con una concepción del mundo en la que el ser humano forma parte del entorno natural y social. Ya lo señalaba Dewey (1961): "la relación existente entre democracia y educación es recíproca, mutua, y esto de una manera vital. La democracia constituye en sí misma un principio educativo, un modelo y una forma de educación" (p. 42)

En este sentido, la formación de los futuros docentes deberá girar sobre la base de una visión humanista y democrática de la educación, en la que el profesor tiene una misión de responsabilidad social con sus estudiantes, realizada desde un enfoque que considere a los jóvenes educandos como individuos sujetos de su historia, capaces de transformar su realidad próxima y, colectivamente, aspectos relevantes de la sociedad, en particular la educación. Ello se logrará a partir de la integración con otros individuos en 
su territorio y comunidad escolar, en un ejercicio comunitario, en un proceso de producción y recreación cooperativa de su propio conocimiento, del despertar de su conciencia personal, conectando su existencia e identidad con otros sujetos, en una relación de diálogo. Esta es una experiencia que no se da por sí sola, o a raíz de la preparación para rendir una prueba de contenidos de una asignatura, sino que dentro de una problematización y una visión crítica del contexto social, político y económico que a los jóvenes corresponde vivir (Freire, 1972).

Es necesario que las universidades orienten su proyecto de formación inicial de profesores en concomitancia con los perfiles de egreso, que formen profesionales de la educación con responsabilidad social ciudadana de acuerdo a lo que prescribe esta ley. Con ello, se debe lograr la transformación de los jóvenes y su contexto, dando término al modelo tradicional de enseñanza correspondiente a la concepción bancaria, como lo plantea Paulo Freire en sus escritos (1972). En este enfoque, los individuos son considerados un depósito que recibe pasivamente cierto tipo de contenido cultural hegemónico, que ha sido digerido por otros previamente. Esa es una práctica atentatoria a una ciudadanía que debe girar en torno a la reflexión crítica, participación activa y discusión del tipo de sociedad que se busca construir, aprovechando el espacio de la sala de clases para integrar los diferentes temas de interés general y estudiantil, práctica que ciertamente los jóvenes esperan realizar a partir de los diferentes enfoques que representan las asignaturas del currículum.

La formación ciudadana debe complementar las definiciones curriculares, lo que representa para las escuelas un importante desafío para que sus Proyectos Educativos Institucionales (PEI) replanteen su micropolítica, incorporando este enfoque desde la diversidad de prácticas cotidianas realizadas por los jóvenes. Esta experiencia nace de un ejercicio contrahegemónico respecto del imaginario de país en que desean vivir, el cual no se condice con el academicismo característico del modelo educativo que busca homogeneizar y no considerar el valor de lo que significa la práctica 
de la cultura juvenil (Álvarez, 2016). Esta mirada de país, construida por la cultura hegemónica adultocéntrica, nace desde una mirada cultural, social y de acción política realizada en sus respectivos territorios, la que se destaca por generar espacios de ciudadanía autónoma y autogestionada, realizada por fuera del sistema político del cual no se sienten parte por encontrarse desacreditado (Álvarez, 2016).

Por ello, la escuela tiene el desafío de visibilizar a los jóvenes no solo como estudiantes sino como ciudadanos que efectivamente puedan construir ciudadanía desde la reivindicación de sus derechos y desde la autonomía, la identidad, el territorio y el proyecto de vida, convirtiéndose en sujetos críticos y propositivos y conduciendo a la escuela a convertirse realmente en una institución transformadora mejorando su propuesta educativa (Álvarez, 2016; Flanagan y Levine, 2015; PNUD, 2016).

La ley 20.911 apunta a la formación de ciudadanos que asuman una vida responsable, principio común vinculado al ordenamiento jurídico nacional y la institución general del derecho, lo que se relaciona con un atributo de la personalidad que permite al ser humano ser titular de relaciones jurídicas (Ducci, 2005). La responsabilidad en el ordenamiento jurídico chileno nace de la concepción de persona humana, definida desde una doble dimensión, tanto moral como civil (Pacheco, 2004). Desde un punto de vista moral, se entiende por "persona humana" un sujeto que se encuentra dotado de razón y voluntad, que es libre para aspirar a los fines que estime convenientes. Por otro lado, civilmente, se entiende por persona humana al sujeto de derechos, capaz de adquirir derechos y contraer obligaciones jurídicas ( $\mathrm{Pa}-$ checo, 2004). Al constituirse como persona por la razón y libertad individual, se aspira a derechos y obligaciones al interior de un colectivo, lo cual requiere un reconocimiento por parte de los miembros de la comunidad.

Desde un punto de vista del derecho civil, uno de los atributos de la personalidad del ser humano es su capacidad de goce y ca- 
pacidad de ejercicio (Ducci, 2005), particularidades inherentes a todos los sujetos que la tienen por el solo hecho de nacer, sin exclusiones (Ducci, 2005), salvo la última cualidad que es reconocida para la mayoría de edad ${ }^{17}$, en la que se logra un cierto nivel de aptitud mental y moral, expresada en la adquisición de derechos que sería jurídicamente relacionada con la capacidad de goce. La capacidad de ejercicio es aquella aptitud legal que permite ejercer derechos y contraer obligaciones por sí mismo, sin la autorización de otra persona (Ducci, 2005). La regla general es que todos los sujetos poseen capacidad de ejercicio, existiendo algunas excepciones, como el de los menores de edad, incapaces relativos de contraer obligaciones en tanto se busca proteger sus intereses, pudiendo actuar con autorización de un tutor. De acuerdo a los rangos de edad de la juventud -que define este texto- hay jóvenes con y sin derechos autónomos desde el punto de vista de sus obligaciones y responsabilidad sobre las consecuencias de sus actos. Así, hay juventudes y juventudes en la juventud.

En síntesis, una persona es un sujeto de derechos y de obligaciones en consideración por el solo hecho de ser persona, de ser reconocido por otros miembros de la comunidad como dueño de sus razonamientos y decisiones y por ser miembro de una entidad libre. De aquí nacen los derechos arraigados en la espiritualidad y libertad del ser humano, su sentido de trascendencia y el orden valórico que constituye su identidad.

Cuando todos estos derechos se encuentran con el reconocimiento y respeto de los demás miembros de la comunidad, nos enfrentamos al cumplimiento de los deberes, los que surgen por el sentido de pertenencia y de compromiso con esa comunidad. El cumplimiento de los deberes tiene mucho que ver con el sentido de solidaridad y compromiso con cada uno de los miembros de la comunidad a la que pertenecemos, sea por nacimien-

17 En Chile, a los 18 años se reconoce la ciudadanía, de acuerdo al ordenamiento constitucional. 
to o por afinidad, al entender que la realización de los derechos de los otros contribuye al enriquecimiento de los derechos propios, todo lo cual redunda en la construcción del bien común. Por tanto, no nos preocupamos por el ejercicio de los derechos propios, sino que velamos también por la necesidad de que los otros hagan uso efectivo de sus derechos (Pacheco, 2004; Camps y Giner, 2004).

La responsabilidad es uno de los valores capitales para el fortalecimiento de la democracia, que necesita ciudadanos activos y participativos, con actitudes cívicas para el buen convivir, pero también como característica moral de los individuos (Camps y Giner, 2004), sobre todo para hacer frente al actual contexto de nuestra sociedad en el que se sobrevalora al mercado como mecanismo de equilibrio económico y social.

Hay dos nociones de desarrollo que enmarcan la acción ciudadana y la formación de la ciudadanía en general, y sobre todo respecto de los jóvenes; dos nociones que no se oponen entre sí, sino que se articulan como parte de un mismo proceso político, social, cultural y conceptual de formación: las de desarrollo sostenible y de desarrollo humano.

La UNESCO, en sus "Metas del Objetivo de Desarrollo Sostenible 4: Metas de Educación 2030 para un Desarrollo Sostenible" (UNESCO 2017a, UNESCO 2017b), plantea, en su punto 4.7, una serie de orientaciones referidas al proceso de formación ciudadana que debe impulsar la educación:

De aquí a 2030, garantizar que todos los alumnos adquieran los conocimientos teóricos y prácticos necesarios para promover el desarrollo sostenible, entre otras cosas mediante la educación para el desarrollo sostenible y la adopción de estilos de vida sostenibles, los derechos humanos, la igualdad entre los géneros, la promoción de una cultura de paz y no violencia, la ciudadanía mundial y la valoración de la diversidad cultural y 
de la contribución de la cultura al desarrollo sostenible, entre otros medios ${ }^{18}$.

En ese contexto integral, mundial y cultural, la vivencia del compromiso social, como responsabilidad activa en las transformaciones territoriales, por parte de los jóvenes, es muy importante. No hay plena acción política o social responsable, no hay desarrollo humano con toda su potencialidad real si no es un contexto de sostenibilidad de los territorios, concebidos éstos como territorios de aprendizajes interculturales, donde las personas, la naturaleza, la espiritualidad aprenden, construyen y forman parte de una comunidad de seres vivos que se identifican y relacionan bajo principios de biodiversidad y diversidad cultural (Williamson, 2017) y que, política, cultural y socialmente transforman el mundo.

\section{Juventud y compromiso ciudadano de un modo socialmente responsable}

La Ley de Formación Ciudadana instalada en las escuelas, y por tanto en la sociedad, se asocia a un concepto de desarrollo de los territorios que sitúa al ser humano como parte del mundo en el centro de sus propósitos, objetivos, políticas y acciones.

En la sociedad actual se busca dar respuesta a distintas preguntas por medio de distintos debates sociales, uno de ellos -paralelo al desarrollo sostenible sin contradecirlo- es sobre el Desarrollo Humano, propuesta elaborada por el Programa de Naciones Unidas para el Desarrollo (PNUD). Este paradigma supera una noción individual, de crecimiento económico, de políticas y resultados sociales según la institucionalidad formal, de invisibilización de los propósitos humanos, democráticos y sustentables del desarrollo.

El concepto de Desarrollo Humano supone la expresión de la libertad de las personas para vivir una vida prolongada, salu-

18 Ver sitio: http://unesdoc.unesco.org/images/0024/002472/247286S.pdf 
dable y creativa; perseguir objetivos que ellas mismas consideren valorables; y participar activamente en el desarrollo sostenible y equitativo del planeta que comparten. Las personas son los beneficiarios e impulsores del desarrollo humano, ya sea como individuos o en grupo ${ }^{19}$.

Según el Informe de Desarrollo Humano sobre la politización en Chile del PNUD, existen ciertas decisiones que deben ser tomadas en conjunto, socialmente, en un proceso que denomina "politización” (PNUD, 2015). El concepto alude a generar conciencia en torno a un determinado tema que antes no estaba en el debate público, lo que traspasa los límites de la política como espacio de ejercicio efectivo o deseado de poder: es "un proceso en curso conformado por distintos actores y momentos, donde cada uno contribuye a la redefinición de aquello que puede ser socialmente decidido" (PNUD, 2015, p. 29).

En este contexto, las instituciones adquieren gran importancia y responsabilidad, debido a que deben dar respuesta efectiva a la complejidad que significan estas demandas, legitimándose ante esta nueva realidad; deben hacerlo con prácticas renovadas que persigan el desarrollo humano, no solo individual, sino también colectivo, en el que los sujetos resulten ser sus protagonistas por medio de sus propias capacidades y organización. Por tanto, "la politización es una condición del Desarrollo Humano porque contribuye a la consolidación de la democracia, ampliando el espacio de participación y libertades políticas, y aportando a la definición de un régimen político y social acorde con las necesidades y expectativas de las personas" (PNUD, 2015, p. 56).

En los últimos años se evidencia un creciente malestar social general, y de los jóvenes en particular, lo que comprende distintas interpretaciones; sin embargo, yendo más allá de éstas, se está ante una demanda de cambios profundos (lo que supera la respuesta a

19 Ver el sitio: http://www.cl.undp.org/content/chile/es/home/ourwork/Desarrollo Humano/in_depth/ 
un simple malestar), pues dicha desazón se ha naturalizado entre los jóvenes. Hoy se ha vuelto a desnaturalizar y, más aún, se ha problematizado, dando cuenta de la desconfianza y descontento con las instituciones de la democracia y su operar -como vimos antes-, expresando una crisis de representación, entre otros problemas con los significados atribuidos a la realidad, que es significada como de exclusión, abuso y de falta de libertades.

En concordancia con este descontento, se ha evidenciado un incremento de manifestaciones de carácter público y plural, social e ideológicamente, y por ende de la participación de la sociedad en el ámbito público, en el cual los jóvenes, respondiendo a sus distintas realidades, se han sentido responsables de hacerse cargo tanto de demandas territoriales como de derechos sociales. Sin embargo, más allá del aspecto cuantitativo, expresado en las cifras de incremento de número, magnitud y diversidad de las movilizaciones, es preciso analizar aspectos cualitativos detrás de las demandas exigidas.

La relevancia del proceso de politización se expresa en el surgimiento de nuevos movimientos sociales, que tienen como protagonistas, en muchos casos, a jóvenes. Estos se perciben a sí mismos en estrecho vínculo con la sociedad, lo que en el marco de la politización se denomina "subjetivación política", siendo el resultado de la interacción entre individuos, instituciones y actores sociales. Estos últimos son claves para el desarrollo humano, ya que son reflejo de las individualidades y de la sociedad de la que forman parte, con el espacio público como campo de sus prácticas y expresión de sentidos.

Para referirse a una politización que promueva el desarrollo humano, es necesario precisar límites. En primer lugar, debe tratarse de prácticas que respeten los derechos humanos (considerando el contexto democrático). En segundo lugar, la participación de los actores sociales no se circunscribe únicamente a lo determinado por la política formal. Y, en tercer lugar, se busca legitimar las distintas posturas existentes en el debate. 
Respecto de la participación política, el PNUD (2015) señala que se evidencia un proceso en el cual la política formal se abre a la sociedad, estableciendo la diferencia que existe entre "lo político" y "la política". Lo primero define todo aquello que la sociedad establece como susceptible de ser decidido colectivamente, abarcando un amplio margen; "la política" es la expresión institucionalizada de un determinado estatus de "lo político". Así, entonces, "la política" sería solo una parte integrante de "lo político". En este sentido, "lo político" difícilmente podría no ser parte de la vida diaria de las personas.

Según sugiere el PNUD (2015), existen seis tipos de involucramiento de actores sociales -como los jóvenes- con "lo político":

- Los comprometidos: personas que presentan altos niveles de interés por los temas públicos, de acción colectiva y de participación institucional. Es un grupo más bien crítico de la sociedad y con una fuerte demanda de cambios.

- Los involucrados individualmente: personas que presentan altos niveles de interés por los temas públicos y de participación institucional. Es un grupo con menor demanda de cambio y de malestar.

- Los ritualistas: personas que presentan altos niveles de participación institucional. Es un grupo que presenta gran respeto por la autoridad y escasa tolerancia al conflicto.

- Los colectivistas: personas que presentan altos niveles de acción colectiva e interés por los temas públicos, pero menor interés en la política formal.

- Los observadores: personas que presentan interés por los temas públicos, pero que no se traduce ni en acciones colectivas ni en participación institucional.

- Los retraídos: personas que presentan bajos niveles de interés por los temas públicos, por la acción colectiva y por la participación institucional. Es un grupo que presenta el mayor nivel de malestar con la sociedad y a la vez es muy poco tolerante al conflicto. 
De acuerdo con estos elementos, se realizó una investigación basada en un estudio de caso a seis jóvenes de la comuna de Carahue, que da cuenta de algunos factores que inciden en la conformación de actores sociales juveniles comprometidos socialmente. El aporte realizado por los entrevistados releva la función del territorio, porque en él identifican, de forma unánime, a la familia, la escuela y las organizaciones sociales contenidas en el territorio como sus referentes de formación. El territorio es entendido como el espacio natural donde hacen sinergia distintos factores para hacer comprometerse socialmente a los jóvenes de la comuna de Carahue.

La familia, a pesar del pesimismo de Beck (2002), que menciona que ya no tiene la misma fortaleza que antes, puesto que la mujer salió del espacio hogareño (división por género del trabajo) y se rompe esa estructura básica. Tanto mujeres como hombres son partícipes del mundo laboral, lo que debilita a la familia; sin embargo, la importancia de los hijos, señala Beck, es la que mantiene o aumenta su importancia. Lo que se condice con lo planteado por Bourdieu (1998), ya que la base del capital cultural tiene sus cimientos en la familia. Sin embargo, debido a los procesos de individualización en la modernidad reflexiva, ha cambiado la configuración de las familias y hoy existen en una gran variedad. La familia es el primer escenario de socialización y formación en el lenguaje materno, en los modos de pensar, sentidos de vida, creencias y valores sociales y políticos.

Otro de los campos de vivencias de esas experiencias con objetivos de formación y desarrollo humano, es el sistema escolar. La escuela para Bourdieu (1998) es un ente formador de gran importancia, pues en este lugar el estudiante desarrolla determinadas formas de pensar y afronta distintas situaciones. Es donde se comienzan a asumir liderazgos y no solo en el Centro de Alumnos, como menciona uno de los entrevistados. M1 dice:

...El colegio es donde está la fuente de información, donde uno forma grupos de amigos y a pequeña escala se van organizando y 
después a medida que uno va creciendo se va formalizando más, se van hablando de temas más de grandes, internamente uno va teniendo más responsabilidades y uno va motivando a otras personas a participar...

M2 por su parte señala:

... a mí me tocó el pingüinazo $0^{20}$ y eso me marcó, fue algo histórico, hasta eso me conmovió, sobre todo porque trataron de frustrarnos, pero pusimos las bases para lo que hoy es la educación gratuita también, demostramos que se puede...

En estos sucesos, entre la situación social del estudiante y su entorno, cobra vida el valor del capital cultural que posee el sujeto, específicamente aquel heredado de la familia, como primera instancia, sumado a las habilidades que transmite la escuela (considerando todos los factores que ella comprende): son lo que determina de cierta forma al sujeto.

Bourdieu (1998) se refiere al habitus, como un lugar específico (territorio, decimos) en el cual se internalizan estructuras mentales, de orden social, que permiten actuar en el mundo generando prácticas sociales características, que, en algunos casos, como en el de este trabajo, apuntan a la escuela, ya que todos los entrevistados mantienen un principio generador y unificador aprendido en el territorio escolar, que retraduce las características intrínsecas y relacionales de un conjunto unitario de nociones respecto de la elección de personas, de bienes y de prácticas, que son moldeados por ella, pero que también dependen de factores propios de este espacio, como ciertos profesores o asignaturas, produciendo efectos inesperados no siempre previsibles.

20 Movimiento de carácter nacional, de estudiantes principalmente secundarios, que en 2006 lograron que se dictara la Ley General de Educación, N 20370, 12.09.2009, que generaba algunas modificaciones a la Ley Orgánica Constitucional de Enseñanza (Ley 18.962, 07.03.1990), dictada por la dictadura militar tres días antes de que asumiera el gobierno de transición democrática. 
De igual forma la politización es un tema recurrente. Una entrevistada identifica un corte en la escuela M2:

La escuela tiene una función transformadora, por lo mismo siento que es un espacio tan importante que se pierde, tener niños y niñas que sepan de sus derechos $y$, claro, de sus deberes, pero es tan importante formar buenos niños y los agobiamos con tareas que pueden aprender en cualquier momento de la vida, no asi otras cosas como principios que nos forman para toda la vida...

Y esto es importante, ya que, según Marshall y Bottomore (1998), en la actualidad existe una disociación entre los derechos sociales, políticos y civiles; por ende, no se puede gozar de una ciudadanía plena cuando estos derechos están aislados unos de otros, perdiéndose un elemento vital para que existan personas responsables, comprometidas con la sociedad: la unidad de las dimensiones y espacios de ejercicio de sus derechos en el mundo y escuela.

Organizaciones sociales; Juárez (2013) plantea que las organizaciones sociales son el motor del desarrollo humano local, porque se centran en el ser humano y en los intereses colectivos de una comunidad, en la que se van potenciando capacidades y la organización de ésta, poniendo el énfasis en el trabajo colectivo para empujar a un territorio determinado hacia mejores condiciones de vida. Así lo plantea la entrevistada M2:

Las organizaciones sociales me siguieron formando absolutamente y ahí la iglesia me entregó muchos más elementos, adquirir herramientas y tener más redes y eso no se va enfocando solo en la iglesia o en el lado espiritual, sino que también eso se utiliza para otras cosas... en el mismo trabajo...

Es decir, lo local es el ámbito en el que la gente vive, la comunidad, en la que las personas se relacionan con el medio y donde se crean los poderes sociales. El territorio como espacio natural, comenta Romero (2009), desde una materialidad, con existencia social, sienta sus bases en la incorporación que hacen sus habitantes y comunidades a la geografía, como también el aporte que 
hace el componente histórico, entendiendo que estos dos factores lo hacen completamente dinámico, con sus consecuencias en las culturas.

Sin embargo, al analizar la influencia de las instituciones en la conformación de actores sociales, hay una crítica que no solo se expresó a través de la literatura expuesta, sino que también se condice con lo recogido de los entrevistados, que son a quienes se culpa de esa desafección y desinterés juvenil en la política. Siguiendo a Beck (2002), el problema radica en que, en vez de adaptar las instituciones a las nuevas realidades generadas por la globalización y transformación mundial de la vida social, se crearon instituciones que buscaron modificar la realidad, generando con ello un distanciamiento entre los intereses y necesidades de los jóvenes con la institucionalidad de la democracia, del Estado y de la sociedad en su conjunto. Al mismo tiempo los entrevistados, unánimemente, dicen desconocer tratados y organizaciones internacionales sobre la juventud, con lo cual también se produce un distanciamiento con la institucionalidad, conquistas sociales y mundo global.

mmm, no, solo conozco sobre los derechos del niño, o de las mujeres, pero nada en torno a juventud, no sé qué se está hablando de la juventud en el mundo.

A nivel local, los entrevistados conocen al Instituto Nacional de la Juventud (INJUV), pero como tienen una evaluación negativa de las instituciones públicas, también no hacen una buena evaluación de él al integrarlo de modo indiferenciado en el conjunto del aparato del Estado.

No obstante, sí valoran al municipio como un espacio cercano, en el que, con responsabilidad, cumpliendo, pueden sacar el estigma que existe sobre la juventud y, después de dar pruebas de confianza a su contraparte (municipio), se puede realizar grandes alianzas y trabajo efectivo.

Las instituciones deben ser un aporte al fortalecimiento tanto de los actores sociales como de ellas mismas para aportar al desarro- 
llo local. Según Romero (2009), la construcción de lo local es el resultado de un proceso de apropiación de un grupo social, lo que significa una dimensión social y otra material.

Según los entrevistados, los factores que más influyeron en su conformación como actor social comprometido responsablemente con su comunidad fueron la familia, las organizaciones sociales y la escuela. Esto porque, como bien dice Williamson (2002), la participación es una exigencia de la democracia que debe dar respuesta a los tiempos modernos, que emerge de lo social y que lleva al trabajo colectivo. El móvil es buscar el desarrollo económico y social, integrando los principios de los derechos humanos instalados en las luchas sociales de los excluidos, entre éstos, de los jóvenes. Ahí es cuando y donde el desarrollo local humano se fortalece y permite una mejor calidad de vida para todos.

\section{Conclusiones para la educación ciudadana}

El compromiso de los jóvenes con su sociedad depende de los desafíos que cada territorio les exige asumir, desde el espacio local al nacional, o la expresión de lo nacional en el espacio inmediato y próximo al joven, todo ello en una perspectiva de desarrollo humano. No hay responsabilidad sino en relación a un accionar respecto de otros, de contribuir a transformar las condiciones de existencia de éstos y las propias. Los actores juveniles reconocen la educación en los tres espacios de formación señalados en sus trayectorias de vida, la que les lleva a asumir libremente ciertos cometidos, obligaciones, deberes con los demás.

Las experiencias de memoria social, histórica; identidad y etnicidad; religiosidad compartida; vecindad y cercanía geográfica; amistad y compañerismo; relaciones de parentesco; principios y prácticas de reciprocidad, propician el surgimiento de la confianza y la cooperación como elementos articuladores de las organizaciones sociales, que, a su vez, demandan ser los responsables de cambiar la realidad, generando procesos de conciencia que les permiten ser sujetos colectivos de transformación social. 
La familia es un primer espacio educativo, el de la educación informal, asociado a la unidad nuclear de la sociedad, en varias modalidades y por extensión a la familia ampliada e incluso a la comunidad más próxima de los vecinos. Es un momento clave de la socialización, el aprendizaje de la lengua materna, de las cosmovisiones colectivas, de los valores principales ordenadores de la vida social, de las costumbres y relaciones culturales, de la educación democrática y ciudadana desde la palabra y el testimonio. Es el momento inicial en que se va constituyendo una identidad propia según el modo, carácter, significados que se aprenden de las relaciones con los otros, la naturaleza y espiritualidad, en un proceso inacabado que, si bien se inicia como discurso mayor y adulto, se va reconstruyendo -e incluso abandonando a veces- según se experimentan vivencias significativas, particularmente en la etapa juvenil. Se aprende en la reproducción cultural de las decisiones adultas respecto de la infancia que, luego, a medida que pasan los años, se hacen menos rígidas dando paso a una creciente autonomía de los jóvenes respecto de sus propios proyectos de vida; y, en este proceso de desarrollo personal, se va tomando conciencia de los derechos y obligaciones que pueden (o no) irse convirtiendo en acción social o política en la movilización social. No deja de ser común que jóvenes militantes políticos o sociales encuentren en sus familias ambientes favorables a este modo de insertarse en las tareas de cambio social.

La escuela, como segundo espacio o territorio de formación, se asocia a la educación por y para la participación en la construcción de un sentido de comunidad con fines compartidos. Aquí se aprende en la educación formal escolarizada, la socialización secundaria, la formación en el sistema educacional. Este es un sistema formativo que cumple tres funciones claves y contradictorias entre sí: la de la reproducción de la cultura e ideología dominante de la sociedad, la del acceso a partes del conocimiento acumulado por la sociedad y la humanidad, la de generación de conciencia y desarrollo de competencias para la transformación de la sociedad. En esta etapa de la vida, un ambiente de aprendizaje rico 
en experiencias participativas debe generar condiciones para un compromiso juvenil con la sociedad, es decir, un ambiente que no solo prepare para la ciudadanía, sino que la viva. La ley 20.911, que fomenta y garantiza la formación ciudadana, orientada tanto al sistema escolar general, mediante la elaboración de planes en esa materia para todos los establecimientos educacionales del país, como a la formación inicial de profesores en todas las universidades públicas que se especializan en esa materia, reporta un doble desafío, en lo que respecta al proceso de construcción social y política sobre el fortalecimiento civil de la juventud, a partir del foco pedagógico que significa educar en una ciudadanía activa y participativa, más allá de lo que tradicionalmente se ha entendido por lo cívico y la reivindicación de derechos, circunscrita exclusivamente a los mayores de edad.

En primer término, en consonancia con los desafíos propuestos por la globalización y las constantes reivindicaciones sociales, junto con la fuerte mediatización de las relaciones sociales por el acrecentamiento de la actual sociedad-red, es que los jóvenes necesitan ser infundidos con principios de una educación para la ciudadanía activa; estos afectan positivamente el proceso de maduración cognitivo y social para fortalecer el desarrollo psicológico de los estudiantes. Esta línea pedagógica favorece el desarrollo de la responsabilidad social, al formar ciudadanos con habilidades de empatía y comunicación, con responsabilidad personal y social, aportando a la construcción de la personalidad de los jóvenes, contribuyendo a la estimación de la democracia como sistema político y como forma de entablar relaciones sociales.

En segundo término, y como desafío para las instituciones de educación superior, en cuanto a la formación de los futuros docentes, ésta debe girar en torno a cumplir una función de responsabilidad social respecto de su comunidad educativa. Se debe insistir en el paradigma pedagógico de que los estudiantes son el foco central del proceso educativo. Ellos son los sujetos de su historia, los productores y re-productores de su propio conocimiento, capaces de transformar su realidad desde y hacia sus propias comunidades. 
Por eso, los docentes en formación deben recibir habilidades pedagógicas necesarias para orientar a sus estudiantes en los principios de la ciudadanía, tanto para la construcción de la personalidad como para su desenvolvimiento en el compromiso social y democrático. Estos factores deben articularse desde el curriculum escolar, contribuyendo a la formación de una ciudadanía crítica, activa y responsable con ejercicio de derechos y deberes.

El tercer escenario de formación ciudadana son las organizaciones sociales. En la encuesta 2015 del INJUV, un 22,7\% indicó que había participado en movimientos sociales (en baja: el 2012 el porcentaje era de 28,6\%), aunque un 50\% participaba de alguna organización o agrupación, principalmente deportiva $(20,3 \%)$, campañas por internet $(15,3 \%)$, comunidad o grupo virtual $(13,3 \%)$ (a través de redes informáticas, un total de $28,6 \%)$ y en las iglesias, organizaciones religiosas o espirituales (13\%), actividades de ayuda a la comunidad o voluntarias $(28,3 \%)$ (INJUV, 2018a). Los testimonios recogidos por Torres (2017) muestran que esta participación ha sido clave en los jóvenes, y que se levanta en torno a intereses precisos, paralela más que opuesta a formas institucionalizadas de participación: constituyendo un antecedente de formación y compromiso ciudadano relevante en jóvenes militantes sociales y políticos comprometidos con el desarrollo humano local. Otro ejemplo, ahora con jóvenes universitarios, es el de la Universidad de La Frontera (Universidad de La Frontera, 2018) que durante tres años (20162018) no ha contado con Federación de Estudiantes, sin embargo, en el periodo 2013-2017 ha formado 70 agrupaciones estudiantiles libres de las que participan 1.200 estudiantes (p. 174), de ellas 52 generaron 107 actividades solidarias (p. 310). Sin duda que la desarticulación organizacional de la sociedad, producto de la intermediación financiera y fetichista del mercado capitalista entre los seres humanos, ha generado un debilitamiento de la cantidad, calidad y profundidad de las relaciones sociales que se conforman en redes de asociatividad, cooperación y lucha social por una sociedad democrática. 
Lo local y lo temático han asumido un papel relevante en la acción social, un espacio de unidad en torno a necesidades inmediatas $\mathrm{u}$ objetivos específicos de lucha social, ejemplos que pueden apuntar a transformaciones sociales a escalas locales o incluso globa$\operatorname{les}^{21}$, pero que también pueden ser ajustes necesarios al sistema capitalista para que, estableciendo cambios progresivos a escalas locales o temáticas específicas que descompriman presiones sociales, no afecten el modelo de reproducción y acumulación de capitales o al mercado como organizador de la vida social y sus interacciones, o para que, en el tiempo, sean cooptados por nuevos nichos o productos de mercado. Por otra parte, su contribución a la profundización democrática permite construir sociedades que no se centren solo en el crecimiento económico, sino que estén más próximas a un desarrollo humano como organizador de la vida social, concepto que supone participación, gobierno para y por el pueblo, justicia social, igualdad y equidad. No hay ciudadanía sin participación y conciencia, sin organización y dirección social y política: formar a los jóvenes en estas cuestiones es un desafío actual para la sobrevivencia y profundización de la democracia, la libertad, la conciencia social y política, el desarrollo humano. En ello, el sistema escolar y la organización social deben articularse para potenciar, desde dentro y desde fuera del proceso pedagógico, la educación ciudadana de los jóvenes que deben ser los protagonistas de sus propios aprendizajes y de los procesos pedagógicos de cambio social local o global.

\section{Referencias bibliográfica}

Adorno, R. de C. F. (2001). Capacitação Solidaria. Um olhar sobre es jovens e sua vulnerabilidade social. São Paulo, Brasil: AAPCS.

Álvarez, E. (2016). Es necesario visibilizar en los estudiantes no solamente la dimensión de alumnos, sino también la de ciudadanos. Revista Docencia, 58, Colegio de Profesores de Chile.

21 Como el movimiento feminista en Chile y países de varios continentes el primer semestre 2018. 
Argumedo, M. (2011). Lo que se aprende en la escuela. Representaciones básicas en la constitución del ciudadano. Alemania: Editorial Académica Española.

Beck, U. (2002). La sociedad del riesgo global. España: Siglo XXI.

Benítez Z. M. y Echeverría M. (2018). Participación ciudadana y juventud: una aproximación a las actuales formas de participación juvenil, en INJUV (2018) Concurso Nacional de Tesis sobre Juventud. Piensa la Juventud. Resumen Ganadores 2015-2016 (pp. 48-63).

Bourdieu, P. (1998). Capital Cultural, Escuela y Espacio Social. (Trad. I. Jiménez). México. D.F.: Siglo Veintiuno Editores.

Camps, V. y Giner, S. (2004). Manual de civismo. Barcelona, España: Editorial Ariel.

Council of Europe. (2017). Compass, el Manual para la Educación en los Derechos Humanos (EDH) con jóvenes. Recuperado de https://www.coe.int/es/web/compass/citizenship-andparticipation.

Dewey, J. (1961). El Hombre y sus problemas. Buenos Aires, Argentina: Editorial Paidós.

Diccionario de Ciencias de la Educación. (2003). Colombia: Grupo Internacional de Libreros.

Donoso R. A. y Dragnic G. M. (2015) Hacia la universidad pública: aproximación a la importancia del movimiento estudiantil chileno de 2011 en perspectiva latinoamericana, en CLACSO. (2015). Los desafíos de la universidad pública en América Latina y el Caribe (pp. 119-145). Buenos Aires, Argentina: CLACSO.

Ducci, C. (2005). Derecho civil. Parte general. Santiago, Chile: Editorial Jurídica de Chile.

Flanagan, C. \& Levine, P. (2015). Involucramiento Cívico y la Transición a la Adultez, en C. Cox \& J. C. Castillo, Aprendizaje de la Ciudadanía: Contextos, Experiencias y Resultados. Santiago de Chile: Ed. Universidad Católica de Chile.

Flores, J. (2014). Derecho a la Educación: su contenido esencial en el Derecho Chileno. Estudios constitucionales, 12(2), 109136. Recuperado de https://dx.doi.org/10.4067/S071852002014000200005

Freire, P. (1972). La Educación como práctica de la libertad. Buenos Aires, Argentina: Siglo XXI.

INJUV. (2017). Revista RT. Instituto Nacional de la Juventud, 6(24). 
INJUV. (2018a). 8 ${ }^{a}$ Encuesta Nacional de Juventud 2015. Informe Regional. Región de La Araucanía. Santiago, Chile: INJUV.

INJUV. (2018b). Revista RT. Instituto Nacional de la Juventud, 7(25).

Juárez, G. (2013). Revisión del concepto de desarrollo local desde una perspectiva territorial. Revista Líder, (23), 9-28.

Marshall, T. \& Bottomore, T. (1998). Ciudadanía y Clase Social. Madrid, España: Alianza Editorial.

Ministerio de Educación de Chile. (2013). Orientaciones técnicas y guiones didácticos para fortalecer la Formación Ciudadana. $7^{\circ}$ básico a $4^{\circ}$ medio. Santiago de Chile: División de Educación General.

Ministerio de Educación de Chile (2016a). Orientaciones para la elaboración del Plan de Formación Ciudadana. Santiago de Chile: Ministerio de Educación, División de Educación General.

Ministerio de Educación (2016b). Orientaciones Curriculares para el Desarrollo del Plan de Formación Ciudadana. Santiago de Chile: Ministerio de Educación, División de Educación General.

Ministerio de Desarrollo Social. (2016). Encuesta Casen 2006-2015. Situación de la Pobreza en Chile. Santiago, Chile: Ministerio de Desarrollo Social.

Oyarzún, A., Dávila, O., Ghiardo F., Hatibovic F. (2008). ¿Enfoque de derechos o enfoque de necesidades? Modelo de gestión para el desarrollo de un sistema local de protección de derechos de la infancia y adolescencia. Santiago, Chile: Centro de Estudios Sociales-CIDPA, Servicio Nacional de MenoresSENAME.

Pacheco, M. (2004). Teoría del Derecho. Santiago, Chile: Editorial Jurídica de Chile.

Programa de las Naciones Unidas para el Desarrollo, PNUD. (2016). Panorama General. Informe sobre Desarrollo Humano 2016. Desarrollo Humano para Todos. Santiago, Chile: Programa de las Naciones Unidas para el Desarrollo (PNUD).

Programa de las Naciones Unidas para el Desarrollo, PNUD. (2015) Desarrollo Humano en Chile. Los tiempos de la politización. Santiago, Chile: Programa de las Naciones Unidas para el Desarrollo (PNUD).

Romero, H. (2009). Desarrollo local a escala humana. Revista de la Universidad Bolivariana, 8(22), 137-158.

Torres, T. (2017). Conformación de actores sociales juveniles ciudadanos en la comuna de Carahue. Tesis para obtener el grado de 
Magíster en Desarrollo Humano Local y Regional. Universidad de La Frontera, Temuco, Chile.

UNESCO. (2017a). Declaración de Buenos Aires. Reunión Regional de Ministros de Educación de América Latina y el Caribe, 2425 de enero de 2017. Buenos Aires, República Argentina: UNESCO.

UNESCO (2017b) Declaración de Buenos Aires - E2030: Educación y habilidades para el siglo 21. Buenos Aires, Argentina: UNESCO.

Universidad de La Frontera. (2018). Informe de Autoevaluación Institucional UFRO 2018. Temuco: Universidad de La Frontera.

Williamson, G. (2017) Territorios de Aprendizajes Interculturales. Temuco, Chile: Ediciones Universidad de La Frontera.

Williamson, G. (2002). Investigación Acción Participativa Intercultural en Comunidades Educacionales y Locales. Santiago, Chile: Universidad de La Frontera / Proyecto Kelluwün, Temuco. 